\title{
Variant von Willebrand's Disease
}

\author{
CHARACTERIZATION OF TWO SUBTYPES \\ BY ANALYSIS OF MULTIMERIC COMPOSITION OF \\ FACTOR VIII/VON WILLEBRAND FACTOR
}

IN PLASMA AND PLATELETS

ZAVERIO M. RUGGERI and THEODORE S. ZIMMERMAN,
Department of Molecular Immunology, Research Institute of Scripps Clinic,
La Jolla, California 92037

A в S T RACT We have examined the multimeric composition of factor VIII/von Willebrand factor in plasma and platelet lysates by means of sodium dodecyl sulfate agarose electrophoresis followed by staining with ${ }^{125}$ I-labeled affinity-purified antibody. In normal plasma and platelet lysates, factor VIII/von Willebrand factor displayed 10 distinct multimers that ranged in apparent molecular weight from 0.86 to $9.9 \times 10^{6}$. The molecular weight difference between adjacent bands was $0.8-1.1 \times 10^{6}$. Larger material, not resolved into discrete bands, was also present with an average $M_{\mathrm{r}}$ of $14.5 \times 10^{6}$. Though the dimer (apparent $M_{\mathrm{r}}=0.48$ $\times 10^{6}$ ) and the monomer (apparent $M_{\mathrm{r}}=0.28 \times 10^{6}$ ) generated by reduction of disulfide bonds were readily identified in this system, they were not detected in normal plasma or platelets. No differences were observed between fresh plasma prepared without anticoagulant and fresh or frozen plasma anticoagulated with either citrate or heparin. "Variant" (type II) von Willebrand's disease could be divided into two subtypes. In subtype IIA, factor VIII/von Willebrand factor in plasma consisted predominantly of the five smaller multimers with traces of the sixth and seventh $\left(M_{\mathrm{r}}\right.$ up to $\left.4.5 \times 10^{6}\right)$. In subtype IIB, all these multimers were easily detected and, in addition, bands of inter-

Preliminary reports of this work were published in abstract form in 1979. Blood. 54(Suppl. 1): 300a, and in 1980. Fed. Proc. 39: 871. This manuscript is publication number 2007 from the Immunology Departments of the Research Institute of Scripps Clinic.

Dr. Ruggeri is on leave of absence from the Hemophilia and Thrombosis Centre "Angelo Bianchi Bonomi", Policlinico Hospital of the University of Milan, Italy.

Received for publication 14 December 1979 and in revised form 8 February 1980. mediate size $\left(M_{\mathrm{r}}=8.5 \times 10^{6}\right.$ and smaller $)$ were present. In contrast, the multimeric composition of IIB platelet factor VIII/von Willebrand factor was identical to normal, whereas in subtype IIA the larger multimers were absent from platelets as well as from plasma. In subtype IIB, binding of factor VIII/von Willebrand factor to platelets occurred at lower concentrations of ristocetin than required for normal and multimers of smaller size than in normal bound. On the contrary, in subtype IIA, binding was minimal, as was true of normal factor VIII/von Willebrand factor of equivalent size. Thus, physical as well as functional differences in the two subtypes of variant von Willebrand's disease described suggest that different pathogenetic mechanisms underlie the factor VIII/ von Willebrand factor abnormalities in these patients.

\section{INTRODUCTION}

The multimeric structure of factor VIII/von Willebrand factor $(\mathrm{FVIII} / \mathrm{vWF})^{1}$ has been demonstrated by sodium dodecyl sulfate (SDS) electrophoresis of highly or partially purified human (1-4) and porcine (5) material. However, the actual size distribution of FVIII/vWF in plasma is still controversial because some studies (6-8) have suggested that anticoagulation and/or storage at low temperature might be responsible for inducing artifactual aggregation of the FVIII/vWF molecule.

In patients with "variant" (type II) von Willebrand's disease (VWD), however, the larger forms of FVIII/ vWF are decreased in plasma as judged by crossed

\footnotetext{
${ }^{1}$ Abbreviations used in this paper: DTT, dithiothreitol; FVIII/vWF, Factor VIII/von Willebrand factor; SDS, sodium dodecyl sulfate; VWD, von Willebrand's disease.
} 
immunoelectrophoresis (9-11) and SDS agarose gel electrophoresis (12). These patients have a bleeding tendency and decreased ristocetin-induced plateletFVIII/vWF interaction even when the overall concentration of FVIII/vWF in plasma is normal (11). Thus, size distribution has appeared to be an important correlate of function. However, the identification of a subtype (IIB) of variant VWD with heightened ristocetin-induced FVIII/vWF platelet interaction (13) has suggested that variant VWD is not a homogeneous group, and differences in FVIII/vWF structure might be expected among the different patients.

We have now used a new technique for examining the multimeric composition of FVIII/vWF directly in plasma and platelet lysates without intervening purification steps. This technique demonstrates that FVIII/vWF is present in plasma and platelets as a series of polymers and suggests that the protomer has an apparent $M_{\mathrm{r}}$ of $0.86 \times 10^{6}$. Such a protomer would most closely correspond to a tetramer of the basic FVIII/vWF subunit. Moreover, the multimeric composition of FVIII/vWF differs in subtype IIB from other variants of VWD (subtype IIA) studied. This difference is seen both in plasma and in platelet lysates. These results, when taken in concert with ristocetin-induced FVIII/vWF platelet-binding studies, suggest that subtypes IIA and IIB are caused by different underlying pathogenetic mechanisms.

\section{METHODS}

Materials. Ristocetin was purchased from Lundbeck Company, A/S, Copenhagen-Valby, Denmark. Bovine serum albumin (fraction V) was from Sigma Chemical Company, St. Louis, Mo. Rehydratable agarose gel kits and microzonecells model R-101 were from Beckman Instruments, Fullerton, Calif. Dithiothreitol (DTT) and SDS (electrophoresis purity reagents) were purchased from Bio-Rad Laboratories, Richmond, Calif. Urea (ultrapure reagent) was from Bethesda Research Laboratories, Inc., Rockville, Md. Bovine gamma globulins (fraction II) were from Miles Laboratories, Inc., Elkhart, Ind. All other reagents were of the best grade available.

Collection of blood and preparation of plasma. Blood was obtained from clean venipuncture through a 19-gauge needle and collected in plastic tubes containing 0.1 final vol of $3.8 \%$ trisodium citrate. In some experiments, blood was collected in heparin at a final concentration of $5 \mathrm{U} / \mathrm{ml}$. Platelet-poor plasma was prepared by centrifugation at $4,000 \mathrm{~g}$ for $20 \mathrm{~min}$ at $20^{\circ} \mathrm{C}$. Plasma was immediately separated and tested fresh or frozen at $-70^{\circ} \mathrm{C}$ until used. Plasma samples to be analyzed in SDS agarose gel electrophoresis were mixed with 0.1 vol of $10 \%$ SDS immediately before testing. In some experiments, fresh native plasma was prepared by collecting blood directly into a plastic tube with no anticoagulant, followed by immediate centrifugation at $12,000 \mathrm{~g}$ for $2 \mathrm{~min}$, addition of SDS, and electrophoresis. The sample was applied to the gel within 4 min of venipuncture. Addition of SDS to native plasma without anticoagulant prevented visible fibrin formation over a 24-h observation period. Similar samples without SDS showed fibrin formation usually within 20-40 min from venipuncture.
Samples were obtained from the following patients: 4 patients with VWD type I characterized by prolonged bleeding time, reduced ristocetin-induced platelet agglutination, parallel decrease of $\mathrm{FVIII} / \mathrm{vWF}$ procoagulant activity (VIII:C), FVIII/vWF-related antigen (VIIIR:Ag), and FVIII/ vWF-related ristocetin cofactor (VIIIR:RCo) below $0.30 \mathrm{U} / \mathrm{ml}$ (normal values $>0.51 \mathrm{U} / \mathrm{ml}$ ); 8 patients with variant VWD (VWD type II) subtype IIA who showed prolonged bleeding time, abnormal crossed immunoelectrophoretic pattern of VIIIR:Ag (with absence of the larger, less anodic, forms of FVIII/vWF), absent ristocetin-induced platelet agglutination, and unmeasurable VIIIR:RCo as opposed to normal or only slightly reduced levels of VIII:C and VIIIR:Ag; 10 patients with variant VWD subtype IIB, which differed from subtype IIA by demonstrating increased instead of absent ristocetininduced platelet agglutination and measurable VIII:RCo $(0.11-0.75 \mathrm{U} / \mathrm{ml})$. Bleeding time was prolonged in all IIB patients, and the crossed immunoelectrophoretic pattern was indistinguishable from that of IIA patients (Fig. 1). This new subtype of VWD (IIB) has been characterized in a separate study (13). Normal controls were 12 different volunteers of both sexes with no bleeding abnormality.

Purification of FVIII/vWF. FVIII/vWF was purified from cryoprecipitate of fresh frozen plasma by means of agarose gel chromatography, as reported elsewhere (14).

Preparation of platelet-released FVIII/vWF. Blood (16
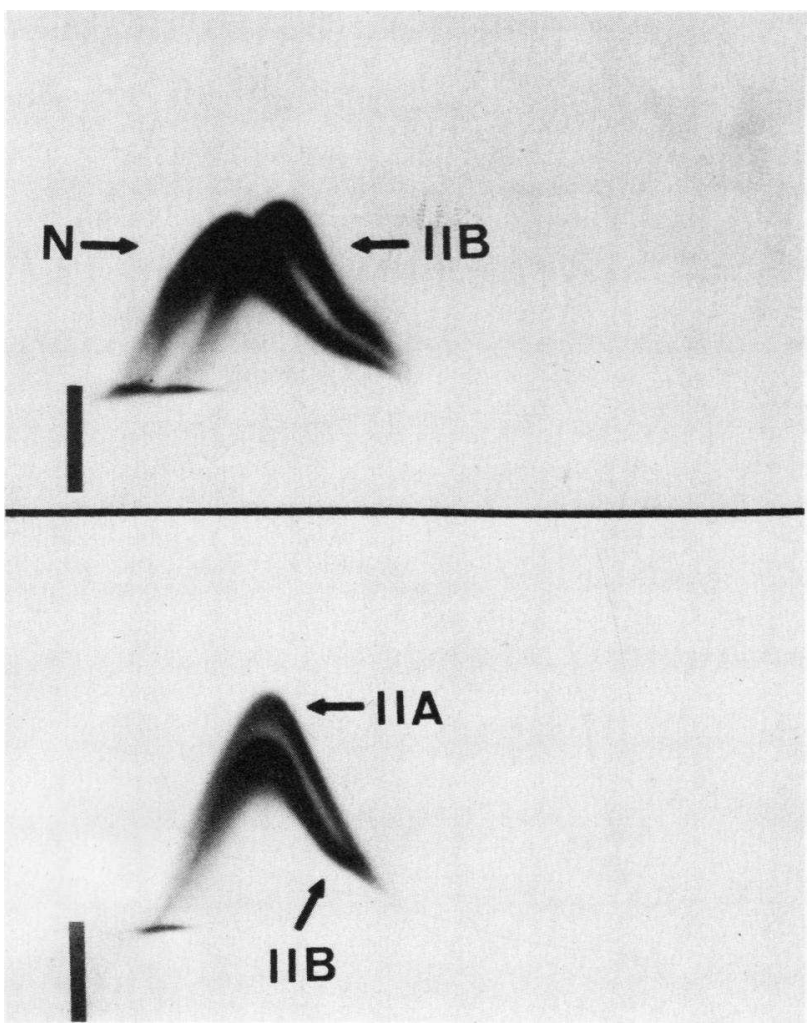

FIgURE 1 Superimposed radiocrossed immunoelectrophoresis autoradiograph patterns of plasma FVIII/vWF. Top panel: normal and subtype IIB superimposed. Bottom panel: subtypes IIA and IIB superimposed. The sample well is indicated by the vertical bar in the bottom left-hand corner of each panel. The anode is to the right in the first dimension and to the top in the second dimension. 
parts) was collected in acid citrate dextrose and platelet-rich plasma was obtained by centrifugation at $120 \mathrm{~g}$ for $15 \mathrm{~min}$ at $20^{\circ} \mathrm{C}$. Platelets were washed four times with the albumin density gradient technique of Walsh et al. (15) and finally resuspended in $0.05 \mathrm{M}$ Tris $-0.1 \mathrm{M} \mathrm{NaCl}$ buffer, $\mathrm{pH} 7.4$. FVIII/vWF was released from washed platelets by means of the hypotonic glycerol lysis technique described by Barber and Jamieson (16), with modifications recently reported in detail (17).

Binding of FVIII/ $\mathrm{eWF}$ to platelets. Binding of plasma FVIII/vWF and platelet-released FVIII/vWF to washed normal platelets was evaluated in the presence of ristocetin. The final suspension of washed platelets had $4 \times 10^{9}$ platelets $/ \mathrm{ml}$ and was prepared in modified calcium-free Tyrode's buffer containing $137 \mathrm{mM} \mathrm{NaCl}, 2 \mathrm{mM} \mathrm{MgCl}, 0.42$ $\mathrm{mM} \mathrm{NaH} \mathrm{PO}_{4}, 11.9 \mathrm{mM} \mathrm{NaHCO}, 2.9 \mathrm{mM} \mathrm{KCl}$, and $5 \mathrm{mM}$ EDTA (tetrasodium salt), $\mathrm{pH} 7.4 .1$ vol of the platelet suspension was mixed with 3 vol of test sample, and then 0.1 final vol of an appropriate solution of ristocetin was added to give final concentrations of $0.5,1,1.5$, and $2 \mathrm{mg} / \mathrm{ml}$. Two control mixtures were tested with each sample, one containing modified Tyrode's buffer instead of platelets (ristocetin concentration in this control mixture was $2 \mathrm{mg} / \mathrm{ml}$ ) and the other containing buffer instead of ristocetin. The mixtures were incubated at $37^{\circ} \mathrm{C}$ for $30 \mathrm{~min}$ without stirring. The supernates were then separated by centrifugation at $12,000 \mathrm{~g}$ for $2 \mathrm{~min}$ and analyzed by SDS agarose gel electrophoresis. Concentration of platelets and incubation time were chosen so that an increase in either did not increase binding even in ristocetin excess.

Reduction of FVIII/cWF. Plasma FVIII/vWF or purified FVIII/vWF were reduced with concentrations of DTT varying between 0.5 and $50 \mathrm{mM}$ by incubation for $2 \mathrm{~h}$ at $37^{\circ} \mathrm{C}$ in the presence of $6 \mathrm{M}$ urea and $1 \%$ SDS.

SDS agarose gel electrophoresis. Electrophoresis was performed with the Beckman rehydratable agarose gel kits. After rehydration according to the manufacturer's instructions, the $0.65 \%$ agarose gels, $1-\mathrm{mm}$ thick, were equilibrated for $30 \mathrm{~min}$ in $0.025 \mathrm{M}$ veronal buffer, $\mathrm{pH} 8.6$, containing $0.1 \%$ SDS. Samples to be analyzed contained $1 \%$ final SDS concentration. A volume of $\sim 1 \mu \mathrm{l}$ was inoculated into the gel by means of the Beckman plastic applicator tip. Up to eight samples could be run on each gel. Electrophoretic separation was obtained by applying a constant current of $9 \mathrm{~mA} / \mathrm{gel}$ for $50-75 \mathrm{~min}$ in a Beckman microzone-cell model R-101. The gel was then fixed for $1 \mathrm{~h}$ in a solution of $25 \%$ isopropanol$10 \%$ acetic acid, washed for $40 \mathrm{~min}$ in two changes of 1 liter distilled water, and soaked for $20 \mathrm{~min}$ in a solution of bovine immunoglobulin (Ig)G (10 mg/ml in veronal buffer), followed by $20 \mathrm{~min}$ in nonimmune rabbit serum $(1-10 \mathrm{vol} / \mathrm{vol}$ in veronal buffer). This last step was found useful in decreasing the nonspecific radioactive background. Finally, the gel was soaked for $14 \mathrm{~h}$ (overnight) in ${ }^{125}$ I-labeled affinity-purified antiFVIII/vWF antibody labeled at a specific activity of $200 \mathrm{mCi} / \mathrm{mg}$ and diluted in $100 \mathrm{ml}$ of veronal buffer to give $\sim 1 \times 10^{6} \mathrm{cpm}$ in $1 \mathrm{ml}$. These antibodies were prepared as described previously (18), with the exception that emus (kindly provided by the San Diego Zoo, San Diego, Calif.), as well as rabbits, were immunized. The gel was then washed in two changes of $0.5 \mathrm{M}$ $\mathrm{NaCl}$ for 4-6 h, in six changes of $0.15 \mathrm{M} \mathrm{NaCl}$ for an additional $24-48 \mathrm{~h}$, dried at $60^{\circ} \mathrm{C}$, and subsequently analyzed by autoradiography using a Kodak XR-1 X-Omat film (Eastman Kodak Co., Rochester, N. Y.) with intensifying screen at $-70^{\circ} \mathrm{C}$. Under the described conditions, a satisfactory autoradiograph was obtained after 5-14 h.

Markers for molecular weight determination. Purified human IgM, prepared by the method of Lawrence et al. (19), was a gift of Dr. Hans Spiegelberg (Scripps Clinic and Re- search Foundation). Human plasma fibronectin, purified according to Mosesson and Umfleet (20), was a gift of Dr. Edward Plow (Scripps Clinic and Research Foundation). Ig.M was polymerized with glutaraldehyde following the procedure described by Fass et al. (5). All of these marker proteins were labeled with ${ }^{125} \mathrm{I}$ at a specific activity of $200 \mathrm{mCi}$ / $\mathrm{mg}$ by a modification of the chloramine $\mathrm{T}$ technique $(21)$. Calibration curves for the determination of molecular weight were obtained in two different ways. For comparison with plasma FVIII/VIVF, one part of the ${ }^{125}$ I-labeled marker was added to nine parts of severe VWD plasma and the mixtures treated like the other plasma samples to be tested. For comparison with purified FVIII/vWF, the ${ }^{125}$ I-labeled markers were used in pure form. Reduced fibronectin was prepared by the method described for the reduction of purified FVIII/vWF The mobility of the ${ }^{125}$ I-labeled markers was evaluated after SDS agarose electrophoresis by direct autoradiography. The marker proteins were always run on the same gel with FVIII/vWF samples.

\section{RESULTS}

Multimeric composition of FVIII/WF in normals. After electrophoresis in SDS agarose gel, FVIII/vWF was separated into multimers of increasing molecular weight. 10 distinct bands, as well as larger unresolved material, were visible in normal plasma on the original autoradiographs. Some resolution was lost with photographic reproduction (Fig. 2). These bands were ' either undetectable in severe VWD plasma or greatly reduced in intensity. There was no difference between fresh native plasma prepared without anticoagulant and fresh or frozen citrated or heparinized plasma (Fig. 2 ). When fresh plasmas without anticoagulant were used, elapsed time from venesection to the end of the

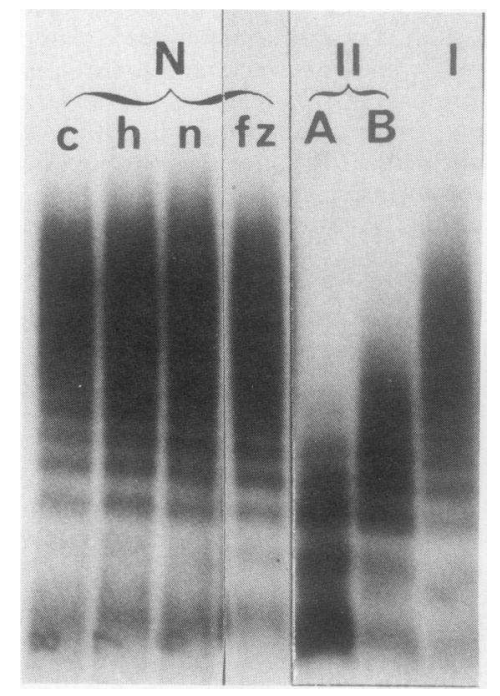

FIGURE 2 SDS agarose gel electrophoresis of plasma FVIII/ $\checkmark W F$ in normal $(\mathrm{N})$, variant (type II) VWD subtypes IIA and IIB, and VWD type I. Normal fresh plasma was either citrated (c), heparinized (h), or native without anticoagulant (n), and was compared with frozen ( $f z$ ) citrated plasma. The point of application is at the top and the anode is at the bottom. 


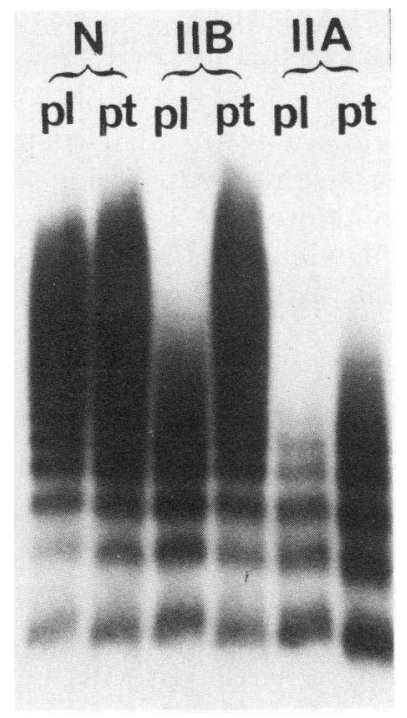

FIGURE 3 SDS agarose gel electrophoresis of plasma ( $\mathrm{pl}$ ) and platelet (pt) FVIII/vWF in normal (N) and variant (type II) VWD, subtypes IIA and IIB. The point of application is at the top and the anode is at the bottom.

electrophoresis was $90 \mathrm{~min}$. During this time, and up to $24 \mathrm{~h}$, no fibrin formation was detectable macroscopically in SDS-treated samples. FVIII/vWF in normal platelet lysates had a multimeric composition identical to that in plasma (Fig. 3). Moreover, purified FVIII/ vWF showed a multimeric composition identical to plasma and platelet FVIII/vWF (Fig. 4). Using unreduced and reduced fibronectin as well as IgM and its aggregates as markers, the apparent molecular weight of the smallest multimer was $0.86 \times 10^{6}$. Multimers of increasing size were separated by a molecular weight interval between 0.8 and $1.1 \times 10^{6}$ (Figs. 4 and 5). The largest material, not resolved into distinct bands, had an average $M_{\mathrm{r}}$ of $14.5 \times 10^{6}$. Mild reduction, (0.05-0.5 $\mathrm{mM} \mathrm{DTT}$ ) of purified FVIII/vWF in SDS urea caused a prompt disappearance of the larger multimers and produced an additional band-presumably the dimer (2) - with apparent $M_{\mathrm{r}}$ of $0.48 \times 10^{6}$ (Figs. 4 and 5). Higher concentrations of DTT $(5 \mathrm{mM})$ reduced all FVIII/vWF to a band-presumably the monomer (2) - with an apparent $M_{\mathrm{r}}$ of $0.28 \times 10^{6}$ (Figs. 4 and 5). Although these latter forms of FVIII/vWF were readily detected in the SDS agarose system by the ${ }^{125}$ I-labeled emu antibody, they were never detectable in unreduced plasma, platelet lysates, or purified material. Rabbit antibodies gave a weak reaction with the dimer but did not react with the monomer. Reduction of plasma FVIII/vWF required about 10 times more DTT than pure FVIII/vWF, but gave the same results.

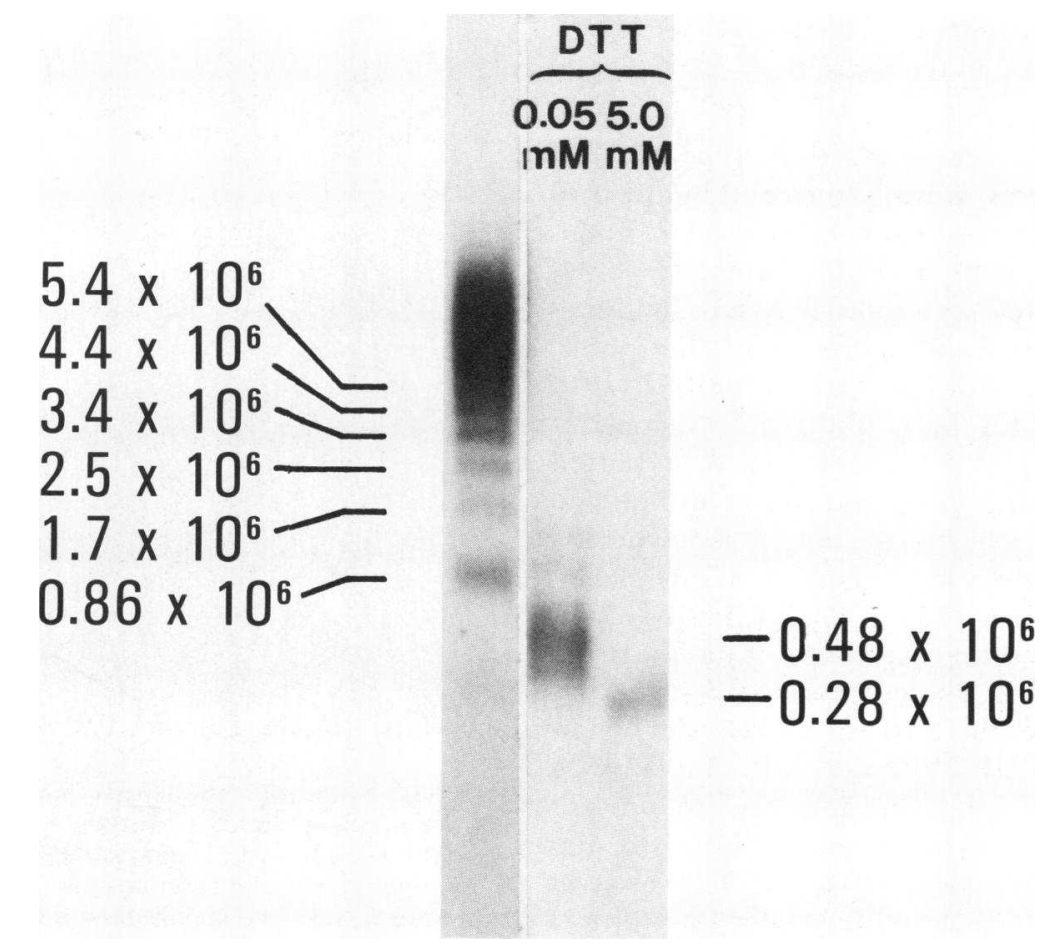

FIGURE 4 SDS agarose gel electrophoresis of purified normal FVIII/vWF, unreduced (left) and reduced with $0.05 \mathrm{mM}$ DTT (middle) or $5 \mathrm{mM}$ DTT (right). Molecular weight is calculated from the calibration curves shown in Fig. 5 . The point of application is at the top and the anode is at the bottom. 


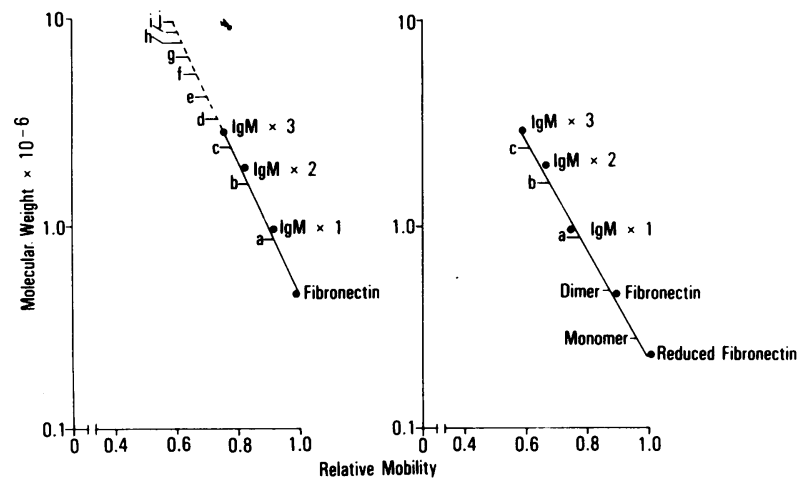

Figure 5 Calibration curves for molecular weight determination of FVIII/vWF multimers in plasma (left panel) or purified fractions (right panel). Left panel: 1 vol of purified marker proteins was mixed with 9 vol of severe VWD plasma before SDS agarose gel electrophoresis. Mobilities of IgM monomer $\left(\operatorname{IgM} \times 1, M_{\mathrm{r}}=0.96 \times 10^{6}\right)$, dimer $\left(\operatorname{IgM} \times 2, M_{\mathrm{r}}\right.$ $\left.=1.92 \times 10^{6}\right)$, and trimer $\left(\operatorname{IgM} \times 3, M_{\mathrm{r}}=2.88 \times 10^{6}\right)$ are expressed relative to fibronectin $\left(M_{\mathrm{r}}=0.66 \times 10^{6}\right)$. The broken line arbitrarily extends the linear calibration curve to $M_{\mathrm{r}}$ of $10 \times 10^{6}$. Horizontal bars $(a-j)$ indicate the relative mobility of $10 \mathrm{FVIII} / \mathrm{vWF}$ multimers of increasing size in normal plasma. Calculated $M_{\mathrm{r}}\left(1^{6}\right)$ are as follows: $\mathrm{a}=0.86$, $\mathrm{b}=1.7, \mathrm{c}=2.5, \mathrm{~d}=3.4, \mathrm{e}=4.5, \mathrm{f}=5.6, \mathrm{~g}=6.6, \mathrm{~h}=7.7$, $\mathrm{i}=8.8, \mathrm{j}=9.9$. Right panel: Purified marker proteins were electrophoresed as such. Mobilities are expressed relative to reduced fibronectin $\left(M_{\mathrm{r}}=0.23 \times 10^{6}\right)$. Horizontal bars indicate the relative mobility of the basic subunit, of its dimer in purified reduced $\mathrm{FVIII} / \mathrm{vWF}$, and $(\mathrm{a}-\mathrm{c})$ of three multimers of increasing size in purified unreduced FVIII/vWF (see Fig. 4).

Multimeric composition of FVIII/wWF in VWD. Two types of patients were studied. In type I VWD, FVIII/vWF-related antigen was decreased below 0.30 $\mathrm{U} / \mathrm{ml}$, but there was no abnormality on crossed immunoelectrophoresis. The patients with variant (type II) VWD all demonstrated abnormal crossed immunoelectrophoretic patterns with absence of the larger, less anodic, forms (Fig. 1). Type II was initially divided into two subtypes on the basis of different ristocetininduced FVIII/vWF platelet interaction. As reported elsewhere in detail (13), little or no ristocetin-induced platelet agglutination occurred in subtype IIA, whereas in subtype IIB agglutination occurred at lower concentrations of ristocetin than required for normals. Investigation of the multimeric composition of plasma and platelet FVIII/vWF showed that these two subtypes were distinct on the basis of the physical characteristics of the FVIII/vWF molecule. In subtype IIA, only the five smaller multimers were present in significant amounts, with traces of the sixth and seventh sometimes detectable (Fig. 2). There was an absolute or relative increase in the smallest multimers as judged by intensity of staining. Platelet FVIII/vWF in this subtype showed small amounts of intermediatesized multimers $\left(M_{\mathrm{r}}\right.$ up to $\left.8.5 \times 10^{6}\right)$, but the largest multimers evident in both normal plasma and normal platelet lysates were not present (Fig. 3). Subtype IIB differed from subtype IIA in that intermediatesized multimers were evident in plasma. The seventh and eighth multimers were easily detectable and, in addition, larger material with average $M_{\mathrm{r}}$ of $8.5 \times 10^{6}$ was usually evident (Fig. 2). A difference between plasma and platelet FVIII/vWF was observed in subtype IIB. The platelet lysates from all these patients displayed an apparently normal multimeric composition (Fig. 3). The findings in these two subtypes of variant (type II) VWD were consistent and reproducible within families and in samples from the same patients obtained at different times. In contrast to type II, type I VWD patients all demonstrated a normal multimeric pattern of plasma (Fig. 2) and platelet $\mathrm{FVIII/vWF}$. Some differences in the relative staining intensity of the smallest multimers were observed in normals and type I VWD patients, but they did not comprise a definite pattern.

Binding of FVIII/ $\mathrm{WWF}$ multimers to platelets in the presence of ristocetin. All but the five smallest multimers of normal plasma and platelet FVIII/vWF bound to platelets at the highest concentration of ristocetin used (Figs. 6 and 7). Concentrations $>2 \mathrm{mg} / \mathrm{ml}$ produced a precipitate that precluded their usage. A preferential binding of the larger multimers was evident with lower quantities of ristocetin. Plasma FVIII/vWF

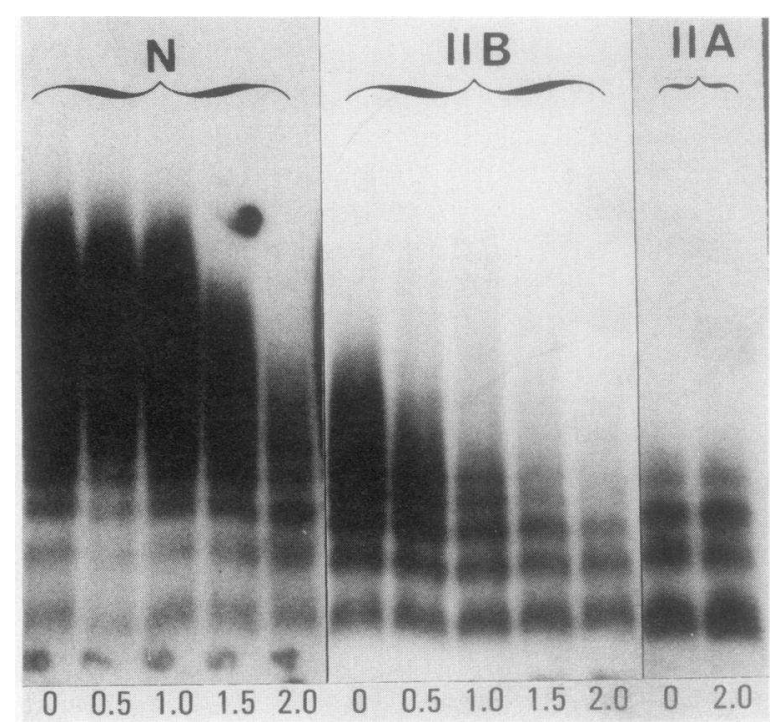

FIGURE 6 Binding of plasma FVIII/vWF multimers to washed normal platelets in the presence of ristocetin. Platelets were mixed with test plasma, and ristocetin was added at the final concentrations (milligrams per milliliter) indicated. After incubation, platelets were removed by centrifugation and the supernates were analyzed by SDS agarose gel electrophoresis (see text for technical details). Plasma from normals (N) and patients with variant (type II) VWD, subtypes IIA and IIB, was analyzed. The point of application is at the top and the anode is at the bottom. 


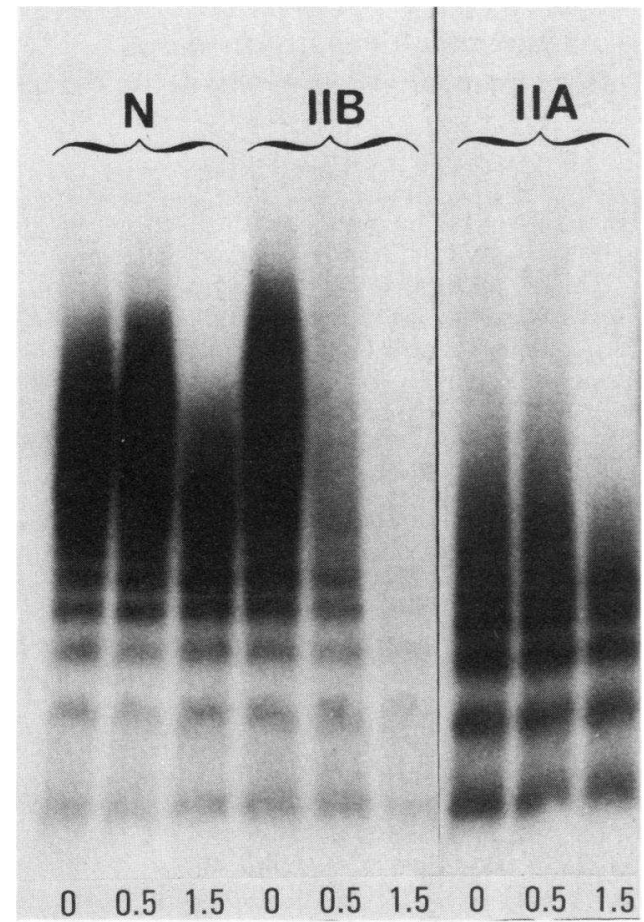

FIGURE 7 Binding of platelet FVIII/vWF multimers to washed normal platelets in the presence of ristocetin (see Fig. 6 for details). Platelet FVIII/vWF from normals (N) and patients with variant (type II) VWD, subtypes IIA and IIB, was analyzed. The point of application is at the top and the anode is at the bottom.

from patients with subtype IIB VWD bound at lower concentrations of ristocetin than required for normal $\mathrm{FVIII/vWF}$, in spite of the absence of the larger molecular forms. In addition, the multimers of IIB FVIII/vWF that bound at any quantity of ristocetin were smaller than the normal multimers that bound at that concentration (Fig. 6). Platelet FVIII/vWF from IIB patients showed a similar enhanced ristocetininduced binding (Fig. 7). As with normal FVIII/vWF, a preferential binding of the largest multimers present was observed at lower quantities of ristocetin. Plasma of patients with subtype IIA VWD contained only the five smallest multimers and these-like the five smallest multimers in normal plasma-showed no appreciable binding at any concentration of ristocetin (Fig. 6). The small amounts of intermediate sized multimers present in type IIA platelets bound in a manner similar to normal platelet FVIII/vWF of the same size (Fig. 7). Thus, FVIII/vWF from subtype IIA, in contrast to that from subtype IIB, showed binding properties similar to normal FVIII/vWF of corresponding size.

\section{DISCUSSION}

Though most investigators are in reasonable agreement concerning the size and multimeric structure of purified FVIII/vWF (1-5), there has been controversy over its molecular weight in plasma. Some authors (6-8) have suggested that the large size in excess of 1 million $M_{\mathrm{r}}$ might result from aggregate formation in vitro as a result of the anticoagulant used and/or the effect of storage and low temperature. The approach we have taken directly addresses this issue because it allows sizing of multimers within $90 \mathrm{~min}$ of venesection, with all manipulations carried out at ambient temperature. SDS - an agent that prevents aggregation-was added within $3 \mathrm{~min}$ of venesection. There was no discernible difference in the multimeric structure of fresh plasma without anticoagulant, fresh heparinized or citrated plasma, and citrated plasma stored at $-70^{\circ} \mathrm{C}$ for $6 \mathrm{mo}$ in the absence of SDS. In addition, platelet FVIII/vWF showed a similar pattern. Therefore, it seems unlikely that the larger multimers are the results of artifacts brought about by processing.

The smallest detectable oligomer of FVIII/vWF in plasma and platelet lysates had an apparent $M_{\mathrm{r}}$ of $0.86 \times 10^{6}$. However, our results have been obtained in the presence of SDS and the possibility exists that these oligomers circulate in native blood as aggregates held together by noncovalent bonds. Mild reduction caused the prompt disappearance of the larger multimers and generated a smaller band of apparent $M_{\mathrm{r}}$ of $0.48 \times 10^{6}$. Complete reduction of disulfide bonds yielded a single band, possibly the FVIII/vWF subunit, with apparent $M_{\mathrm{r}}$ of $0.28 \times 10^{6}$. These results are in agreement with previous findings of Counts et al. (2) and Perret et al. (3) who demonstrated that mild

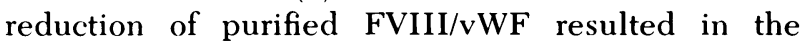
appearance of a band with apparent molecular weight between 0.48 and $0.50 \times 10^{6}$. In comparison with cross-linked FVIII/vWF subunits, they concluded that this was a dimer of the basic subunit and proposed it as the protomer of the multimeric series of FVIII/vWF. However, we found that in plasma and platelet lysates the apparent molecular weight of the smallest oligomer with intact disulfide bonds corresponded to the molecular weight interval between adjacent oligomers, calculated between 0.8 and $1.1 \times 10^{6}$. Therefore, we propose that this oligomer is the protomer of the multimeric series and is a tetramer of the basic FVIII/ $v W F$ subunit. It should be noted that, on the basis of the apparent molecular weight values calculated in this study, the protomer would more closely correspond to a multiple of three subunits. However, it is likely that the size of the subunit is overestimated in SDS agarose (5) or SDS agarose-acrylamide gels $(2-4)$. Values of $0.2 \times 10^{6}$ have been consistently obtained with $5 \%$ acrylamide gels $(22-24)$. Moreover, the assumption that the mobility of an SDS protein complex in SDS electrophoresis is purely dependent on size has a number of exceptions, particularly for glycoproteins and proteins with intact disulfide bonds 
(25-27). Therefore, the comparison of unreduced or partially reduced FVIII/vWF oligomers with other proteins of known molecular weight, as in this study, or with fully reduced cross-linked FVIII/vWF subunits, as previously described $(2,3)$, can only give an approximation of the true molecular weight.

The two subtypes of variant VWD herein described showed abnormal multimeric composition of FVIII/ vWF. This was in contrast with the normal pattern in VWD type I, in which a decreased plasma concentration of otherwise normal FVIII/vWF is likely to be responsible for the hemostatic defect. However, the abnormalities in subtype IIA differed from those in subtype IIB both in plasma and in platelets, suggesting the possibility of distinct molecular abnormalities of FVIII/vWF in variant VWD.

The observed differences in binding of FVIII/vWF to platelets in the presence of ristocetin provide additional evidence that there are intrinsic differences in the FVIII/vWF molecule in these two subtypes. In subtype IIA, the binding properties of the FVIII/vWF present were similar to those of normal FVIII/vWF of corresponding size. Thus, the five smallest multimers in normal plasma or platelets did not bind at any concentration of ristocetin used, nor did they bind from subtype IIA plasma or platelets. On the other hand, subtype IIA platelets contained a small amount of intermediate-sized multimers and this larger material bound as did similar sized material from normal platelets. Subtype IIB FVIII/vWF, on the other hand, showed clear differences from the binding properties of normal FVIII/vWF. Not only did binding require lower concentrations of ristocetin, but at any given quantity of ristocetin the IIB multimers that bound were smaller than the normal multimers binding at that concentration.

These observations, taken with the different multimeric structure of plasma and platelet FVIII/vWF in subtypes IIA and IIB, suggest the possibility of distinct pathogenetic mechanisms underlying the two disorders. Subtype IIA might result from a mutation causing an inability to form the larger multimers. However, the small amounts of intermediate-sized multimers present in platelets appear to bind normally. In subtype IIB, on the other hand, the ability to synthesize the larger multimers would seem to be unimpaired as judged by the analysis of platelet FVIII/vWF. However, there is evidence for a functional abnormality in the FVIII/vWF produced. Assuming that ristocetininduced binding of FVIII/vWF to platelets reflects, to some extent, physiological in vivo binding, it is possible that subtype IIB FVIII/vWF is more rapidly bound to tissue sites and removed from circulation than normal. This rapid removal of the larger multimers would lead to the observed difference in plasma and platelet FVIII/vWF composition. The absence of the larger circulating multimers, as in subtype IIA, would in turn be responsible for the hemostatic defect.

\section{ACKNOWLEDGMENTS}

We wish to thank James Roberts for skillful technical assistance, Roberta Novak for preparation of the manuscript, and the San Diego Zoo for providing emus.

This work was supported by National Institutes of Health grants HL-15491, HL-16411, and HL-20517. Z. M. Ruggeri was supported in part by Fondazione Floriani, Milan, Italy.

\section{REFERENCES}

1. von Mourik, J. A., B. N. Bouma, W. T. laBruyere, S. deGraaf, and I. A. Mochtar. 1974. Factor VIII, a series of homologous oligomers and a complex of two proteins. Thromb. Res. 4: 155-164.

2. Counts, R. B., S. L. Paskell, and S. K. Elgee. 1978. Disulfide bonds and the quaternary structure of Factor VIII/ von Willebrand factor. J. Clin. Invest. 62: 702-709.

3. Perret, B. A., M. Furlan, and E. A. Beck. 1979. Studies on Factor VIII-related protein. II. Estimation of molecular size differences between Factor VIII oligomers. Biochim. Biophys. Acta. 578: 164-174.

4. Weinstein, M., and D. Deykin. 1979. Comparison of Factor VIII-related von Willebrand factor proteins prepared from human cryoprecipitate and Factor VIII concentrate. Blood. 53: 1095-1105.

5. Fass, D. N., G. J. Knutson, and E. J. W. Bowie. 1978. Porcine Willebrand factor: a population of multimers. $J$. Lab. Clin. Med. 91: 307-320.

6. Newman, J., R. B. Harris, and A. J. Johnson. 1976. Molecular weights of antihaemophilic factor and von Willebrand factor proteins in human plasma. Nature (Lond.). 263: $612-613$.

7. Rock, G. A., E. S. Tackaberry, and D. S. Palmer. 1979. Factors affecting the relative distribution of high and low molecular weight forms of Factor VIII. Thomb. Res. 14: 573-587.

8. Seghatchian, M. J., I. M. Nilsson, L. Holmberg, and M. Miller-Andersson. 1979. Molecular size distribution of Factor VIII in native plasma. Thromb. Res. 14: 589-598.

9. Kernoff, P. B. A., R. Gruson, and C. R. Rizza. 1974. A variant of Factor VIII related antigen. Br. J. Haematol. 26: $435-440$.

10. Peake, I. R., A. L. Bloom, and J. C. Giddings. 1974. Inherited variants of Factor-VIII-related protein in von Willebrand's disease. N. Engl. J. Med. 291: 113-117.

11. Gralnick, H. R., Y. Sultan, and B. S. Coller. 1977. Von Willebrand's disease. Combined qualitative and quantitative abnormalities. N. Engl. J. Med. 296: 1024-1030.

12. Meyer, D., B. Obert, G. Pietu, J. M. Lavergne, and T. S. Zimmerman. 1980. Multimeric structure of Factor VIII/von Willebrand factor in von Willebrand's disease. J. Lab. Clin. Med. In press.

13. Ruggeri, Z. M., F. I. Pareti, P. M. Mannucci, N. Ciavarella, and T. S. Zimmerman. 1980. Heightened interaction between platelets and Factor VIII/von Willebrand factor in a new subtype of von Willebrand's disease. N. Engl. J. Med. In press.

14. Montgomery, R. R., and T. S. Zimmerman. 1978. von Willebrand's disease antigen. II. A new plasma and platelet antigen deficient in severe von Willebrand's disease. J. Clin. Invest. 61: 1498-1507.

15. Walsh, P. N., D. C. B. Mills, and J. G. White. 1977. Metabolism and function of human platelets washed by al- 
bumin density gradient separation. Br. J. Haematol. 36: 281-296.

16. Barber, A. J., and G. A. Jamieson. 1970. Isolation and characterization of plasma membranes from human blood platelets. J. Biol. Chem. 245: 6357-6365.

17. Ruggeri, Z. M., P. M. Mannucci, R. Bader, and T. Barbui. 1978. Factor VIII-related properties in platelets from patients with von Willebrand's disease. J. Lab. Clin. Med. 91: $132-140$.

18. Koutts, J., P. N. Walsh, E. F. Plow, J. W. Fenton, II, B. N. Bouma, and T. S. Zimmerman. 1978. Active release of human platelet Factor VIII-related antigen by adenosine diphosphate, collagen and thrombin. J. Clin. Invest. 62: $1255-1263$.

19. Lawrence, D. A., W. O. Weigle, and H. L. Spiegelberg. 1975. Immunoglobulins cytophilic for human lymphocytes, monocytes, and neutrophils. J. Clin. Invest. 55: $368-376$.

20. Mosesson, M. W., and R. A. Umfleet. 1970. The cold-insoluble globulin of human plasma. I. Purification, primary characterization, and relationship to fibrinogen and other cold-insoluble fraction components. J. Biol. Chem. 245: 5728-5736.
21. McConahey, P. J., and F. J. Dixon. 1966. A method of trace iodination of proteins for immunologic studies. Int. Arch. Allergy Appl. Immunol. 29: 185-189.

22. Schmer, G., E. P. Kirby, D. C. Teller, and E. W. Davie. 1972. The isolation and characterization of bovine Factor VIII (antihemophilic factor). J. Biol. Chem. 217: 25122521.

23. Legaz, M. E., G. Schmer, R. B. Counts, and E. W. Davie. 1973. Isolation and characterization of human Factor VIII (antihemophilic factor). J. Biol. Chem. 218: 3946-3955.

24. Shapiro, G. A., J. C. Andersen, S. V. Pizzo, and P. A. McKee. 1973. The subunit structure of normal and hemophilic Factor VIII. J. Clin. Invest. 52: 2198-2210.

25. Pitt-Rivers, R., and F. S. A. Impiombato. 1968. The binding of sodium dodecyl sulphate to various proteins. Biochem. J. 109: 825-830.

26. Dunker, A. K., and A. J. Kenvon. 1976. Mobility of sodium dodecyl sulphate-protein complexes. Biochem. J. 153: $191-197$.

27. Nielsen, T., and J. A. Reynolds. 1978. Measurements of molecular weights by gel electrophoresis. Methods Enzymol. 48: 3-10. 